\title{
ARTICLE
}

Received 28 Dec 2015 | Accepted 6 Jul 2016 | Published 24 Aug 2016

DOI: $10.1038 /$ ncomms12469

OPEN

\section{Assembled molecular face-rotating polyhedra to transfer chirality from two to three dimensions}

Xinchang Wang ${ }^{1, \star}$, Yu Wang ${ }^{1,2}{ }^{\star}$, Huayan Yang ${ }^{1,2}$, Hongxun Fang ${ }^{1}$, Ruixue Chen ${ }^{1,2}$, Yibin Sun ${ }^{1,2}$, Nanfeng Zheng ${ }^{1,2}$, Kai Tan ${ }^{1}$, Xin Lu ${ }^{1,2}$, Zhongqun $\operatorname{Tian}^{1,2} \&$ Xiaoyu Cao ${ }^{1,2}$

In nature, protein subunits on the capsids of many icosahedral viruses form rotational patterns, and mathematicians also incorporate asymmetric patterns into faces of polyhedra. Chemists have constructed molecular polyhedra with vacant or highly symmetric faces, but very little is known about constructing polyhedra with asymmetric faces. Here we report a strategy to embellish a $C_{3 h}$ truxene unit with rotational patterns into the faces of an octahedron, forming chiral octahedra that exhibit the largest molar ellipticity ever reported, to the best of our knowledge. The directionalities of the facial rotations can be controlled by vertices to achieve identical rotational directionality on each face, resembling the homo-directionality of virus capsids. Investigations of the kinetics and mechanism reveal that non-covalent interaction among the faces is essential to the facial homo-directionality.

\footnotetext{
${ }^{1}$ State Key Laboratory of Physical Chemistry of Solid Surfaces and College of Chemistry and Chemical Engineering, Xiamen University, Xiamen 361005, China. ${ }^{2}$ Collaborative Innovation Centre of Chemistry for Energy Materials, Xiamen University, Xiamen 361005, China. * These authors contributed equally to this work. Correspondence and requests for materials should be addressed to X.C. (email: xcao@xmu.edu.cn).
} 
M otifs in architecture, mathematics and nature have inspired chemists to create their molecular counterparts $^{1-9}$. Buckminsterfullerene $\left(\mathrm{C}_{60}\right)^{1}$, for instance, structurally resembles the geodesic dome by Buckminster Fuller. Fuller also conceived of face-rotating polyhedra (FRP) ${ }^{10}$, wherein each face of a polyhedron rotates around its central axis, transferring the two-dimensional (2D) chirality of the faces into the 3D chirality of the polyhedron (Fig. 1a). In mathematics, chirality transfer in FRP is highlighted as an analogous strategy to construct polyhedral dice ${ }^{19}$. In life sciences, some virus capsids can be regarded as $\mathrm{FRP}^{12-15}$, in which each face of the icosahedral cricket paralysis virus capsid contains three quasi-equivalent subunits that form identical rotational directionality (Fig. 1b) ${ }^{14}$. The transfer of 2D chirality of the building block to 3D chirality in FRP through self-assembly can be regarded as a type of emergent property ${ }^{6-18}$.

Inspired by mathematics ${ }^{19}$ and the structures of virus capsids ${ }^{20}$, chemists have synthesized molecular polyhedra with various sizes and shapes through self-assembly. Most of these polyhedra are highly symmetric and achiral ${ }^{20-25}$, and chiral molecular polyhedra with lower symmetry have attracted more and more attention because they increase the complexity of motifs and may bring more sophisticated functions ${ }^{26-31}$. Generally, the construction of chiral molecular polyhedra follows two strategies: to use predetermined 3D chiral building blocks as the edges ${ }^{30,32}$, vertices ${ }^{33,34}$ or faces ${ }^{35,36}$ of polyhedra to transfer 3D chirality of the components to the 3D chirality of the chiral structures, or to use achiral components to generate chiral metal-organic polyhedra ${ }^{37-42}$ during a self-assembly process. The latter approach relates to symmetry breaking, and investigation of the process of such complex molecular self-assembly may help to elucidate the evolution of matter towards complexity ${ }^{43}$. However, metal-organic polyhedra remain the dominant type of chiral molecular polyhedra assembled from achiral components, and the resolution of the mixed racemic structures as well as scrutiny of the intermediates and the formation mechanism are challenging.

Here we report a strategy to assemble a $C_{3 \mathrm{~h}}$-symmetric building block into purely organic FRP (Fig. 1c) through dynamic covalent chemistry ${ }^{44-46}$. The building block loses its $\sigma_{\mathrm{h}}$ mirror symmetry in the polyhedra and brings either a clockwise $(C)$ or an anticlockwise (A) rotation around the centre of each face, thus generating a series of FRP stereoisomers. Our thermodynamic polyhedra exhibit homo-directionality, which resembles the identical rotational directionality of each facial unit in some virus capsids. We also investigated the formation mechanisms of the FRP from rotational facial units based on their high spectral sensitivities. The noncovalent interaction among faces has been suggested to be the primary reason for the facial homo-directionality.

\section{Results}

Synthesis of FRP 1 with achiral vertices. The facial building block TR (Fig. 1c and Supplementary Fig. 1) consists of a rigid planar 10,15-dihydro-5H-diindeno[1,2-a;10,20-c]fluorine (truxene) core ${ }^{47,48}$, six alkyl chains at central positions to increase solubility and prevent aggregation, and three carboxaldehyde groups at peripheral positions for reversible imine formation. This building block was readily synthesised from truxene in three steps with high yields (Supplementary Fig. 2).

The truxene building block (TR) (4 eq) and ethylene diamine (EDA) (6 eq) were reacted in toluene with catalytic amounts of trifluoroacetic acid (TFA) at room temperature, affording $\mathrm{TR}_{4} \mathrm{EDA}_{6}$ octahedron 1 in high yield. The mass spectrum of 1 confirmed that it was formed exclusively in solution and exhibited a molecular weight (M.W.) of 3196.29 daltons (calculated M.W.: 3196.31), corresponding to a $[4+6]$ composition (Fig. 2a).

Single-crystal characterization of FRP 1. Single crystals were grown by diffusing hexane into the toluene solution of $\mathbf{1}$. Single-crystal X-ray diffraction analysis revealed that 1 has a tetra-capped octahedral structure $e^{21,33}$, in which four truxene units occupy four faces linked by six diamine units in the vertices (Fig. 2b). Truxene is achiral in three dimensions because of the presence of a mirror symmetry, and chiral in two dimensions because the three $\mathrm{sp}^{3}$ carbons of the truxene core form a rotating pattern along the $C_{3}$ axis in either a clockwise or an anticlockwise direction (Fig. 1c), similar to the pattern in some virus capsids. Therefore, the truxene fragment loses its mirror symmetry in a polyhedron and becomes a rotational face, resulting in five possible stereoisomers for $\mathbf{1}$; these configurations are named after the directionalities of the exterior faces as CCCC and $A A A A$ (homo-directional with $T$ symmetry), CCCA and CAAA (hetero-directional with $C_{3}$ symmetry) and CCAA (hetero-directional with $S_{4}$ symmetry) a

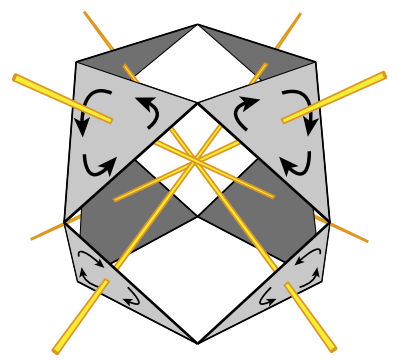

C

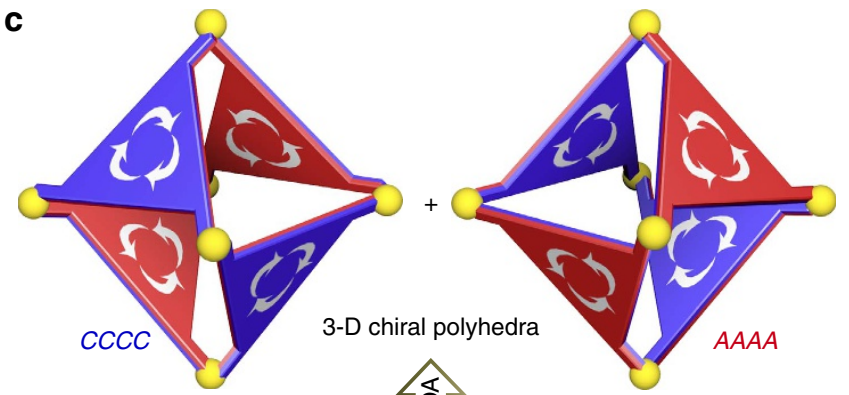

b

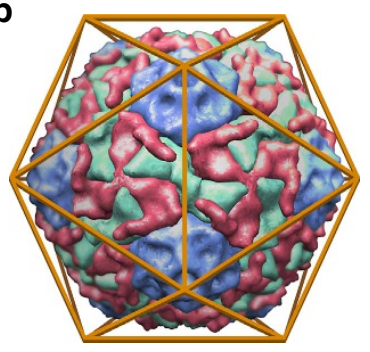

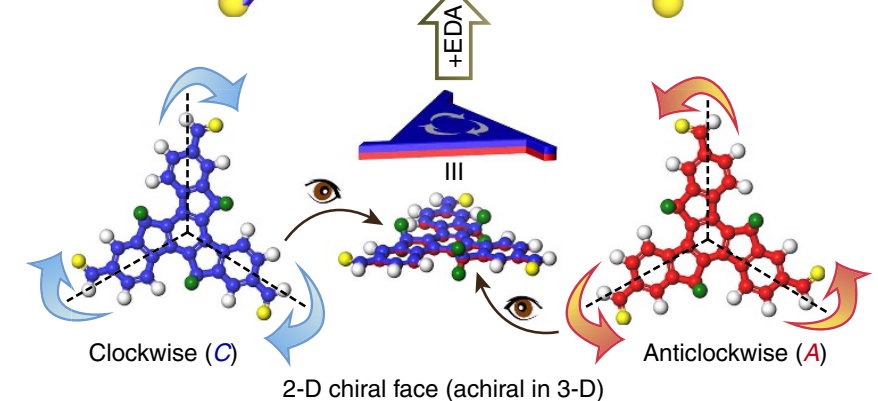

Figure 1 | 3D chiral polyhedra originated from 2D chiral facial units.

(a) Synergetic interactions between the rotating triangular faces in a polyhedron proposed by Buckminster Fuller. (b) All subunits of icosahedral cricket paralysis virus have identical rotational directionality. (c) Truxene building block shown as a ball-and-stick model (red and blue, carbon; white, hydrogen; yellow, oxygen; green, butyl group); its rotation patterns are considered to be either clockwise $(C)$ or anticlockwise $(A)$ when viewed vertical to the aromatic plane. By reacting with ethylene diamine to form an octahedron, the truxene fragment loses its mirror symmetry and becomes a rotational face. 


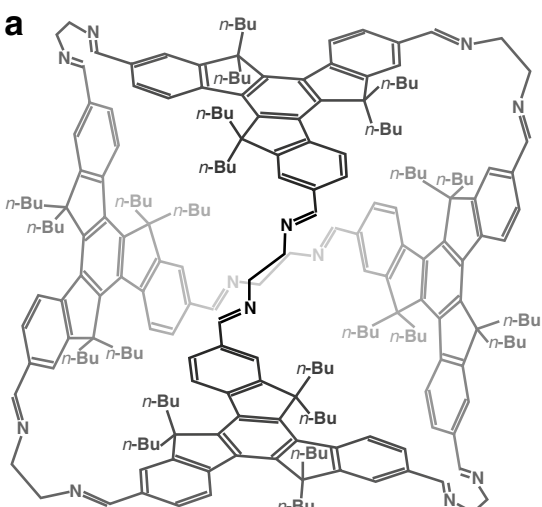

$(C C C C)-1$

C

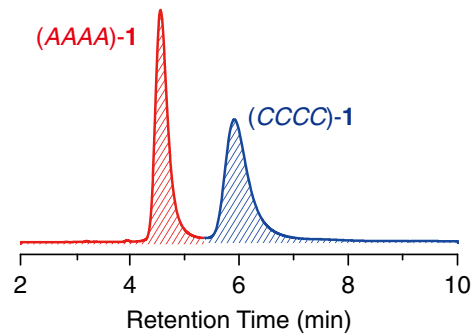

b

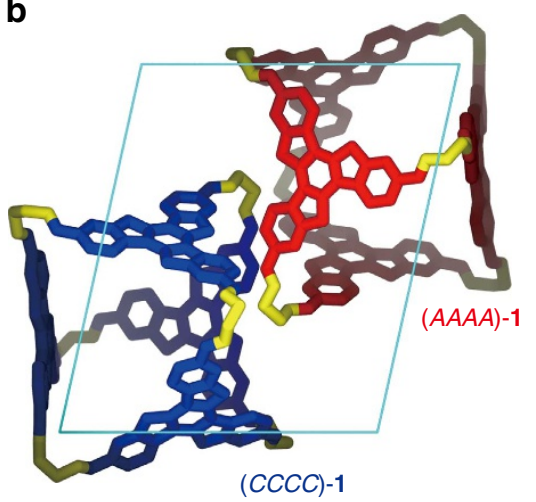

d

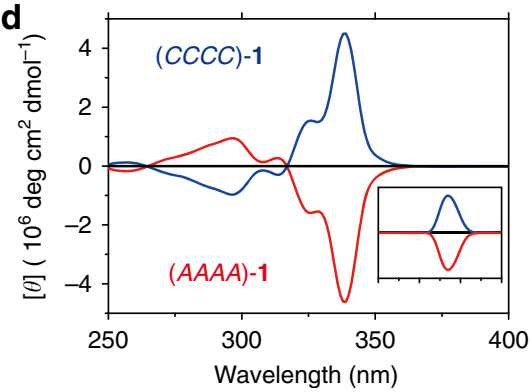

Figure 2 | Molecular structure and characterisation of octahedra 1. (a) Molecular structure of the octahedron (CCCC)-1. (b) Single-crystal structure of the co-crystal of (CCCC)-1 and (AAAA)-1 (hydrogen atoms and butyl groups are omitted for clarity). (c) Chiral HPLC spectrum contains two peaks corresponding to (AAAA)-1 and (CCCC)-1 in a 1:1 ratio. The absorbance for all octahedra was recorded at $325 \mathrm{~nm}$. (d) Experimental and (ZINDO/S)predicted (the insert) CD spectra of ( $A A A A)-\mathbf{1}$ (red), (CCCC)-1 (blue), and the unseparated racemic mixture (black) in hexane.

(Supplementary Fig. 1). We measured more than five single crystals of 1, and found all of them were the co-crystallization of only the $T$-symmetric enantiomers (i.e., (CCCC)-1 and $(A A A A)-1)$ packed in the $P-1$ triclinic space group (Fig. 2b). No other diastereomers or self-sorting of the two enantiomers into two enantiopure crystals was observed. All imine bonds are $E$ conformers, locating in the conjugated plane and rotating in the same direction as the $\mathrm{sp}^{3}$ carbons of the truxene core. The butyl groups compacted inside the octahedra distort the terminal carbons, whereas the butyl groups that stretch outside remain linear.

NMR characterization of FRP 1. The nuclear magnetic resonance (NMR) spectra of $\mathbf{1}$ (Supplementary Figs 3-9) are consistent with its single-crystal structure. The ${ }^{1} \mathrm{H}$ NMR spectrum (Fig. 3) reveals only a single set of peaks for all twelve sets of protons on the truxene backbones $\left(\mathrm{H}^{\mathrm{a}}, \mathrm{H}^{\mathrm{b}}\right.$, and $\left.\mathrm{H}^{\mathrm{c}}\right)$ and imines $\left(\mathrm{H}^{\mathrm{d}}\right)$, suggesting that these protons are individually interchangeable through twelve symmetry operations ${ }^{49}$. Therefore, only the $T$-symmetric stereoisomers of $\mathbf{1}$ exist in solution. All butyl protons inside the octahedron are more shielded from the three neighbouring truxene units than those outside. The 2D NMR spectra allowed for the assignment of each proton and carbon of octahedron 1. In addition, the nuclear Overhauser effect (NOE) crosspeak between imine protons $\mathrm{H}^{\mathrm{d}}$ and $\mathrm{H}^{\mathrm{b}}$ (instead of $\mathrm{H}^{\mathrm{c}}$ ) suggests that all imine bonds rotate in the same direction as the $\mathrm{sp}^{3}$ carbons of the truxene core in solution, and the crosspeak between $\mathrm{H}^{\mathrm{d}}$ and $\mathrm{H}^{\mathrm{el}}$ reflects the shape-persistency and rigidity of the octahedron $\mathbf{1}$ in solution (Fig. 3, insert).
Chiral-HPLC and CD characterizations of FRP 1. Further application of chiral high-performance liquid chromatography (HPLC) and circular dichroism (CD) spectroscopy confirmed that, among all possible stereoisomers, only (CCCC)-1 and $(A A A A)-1$ exist in a 1:1 ratio both in crystal and in solution (Fig. 2c). The solution of crude 1 gives no detectable CD signal. In contrast, the CD signal of the separated octahedron eluting at $4.6 \mathrm{~min}$ gives a strong negative signal in the truxene absorption region $(340 \mathrm{~nm})$, whereas the fraction at 5.9 min provides a mirror-like positive signal (Fig. 2d). The CD signals of both separated octahedra have a molar ellipticity of $4.6 \times 10^{6} \mathrm{deg} \mathrm{cm}^{2}$ $\mathrm{dmol}^{-1}$, which, to our knowledge, is the largest molar ellipticity ever reported ${ }^{50}$ and is substantially larger than that of helicene (ca. $\left.3.5 \times 10^{5} \mathrm{deg} \mathrm{cm}^{2} \mathrm{dmol}^{-1}\right)^{51}$. We attribute this large molar ellipticity to the high molar absorptivity of truxene and to the rigid geometry of the octahedra. The $\mathrm{CD}$ spectrum of the octahedron at $4.6 \mathrm{~min}$ matches the (ZINDO/S)-predicted CD spectrum of $(A A A A)-\mathbf{1}$, and the $\mathrm{CD}$ spectrum at $5.9 \mathrm{~min}$ corresponds to (CCCC)-1 (Fig. 2d, insert).

Synthesis and characterizations of FRP 2 with chiral vertices. After achieving homo-directionality of each face of the molecular polyhedra, we attempted the enantioselective assembly of FRP, similar to the assembly observed in nature for some virus capsids $^{12-15}$. Inspection of the single-crystal structure of $\mathbf{1}$ revealed that all EDA vertices of $(A A A A)-\mathbf{1}$ are in a gauche conformation, and the two amino groups have a torsion angle of ca. $-60^{\circ}$, which is the same torsion angle between the amino groups in $(R, R)$-diaminocyclohexane (CHDA) (Supplementary Fig. 10). Similarly, the amino groups in the vertices of (CCCC)-1 


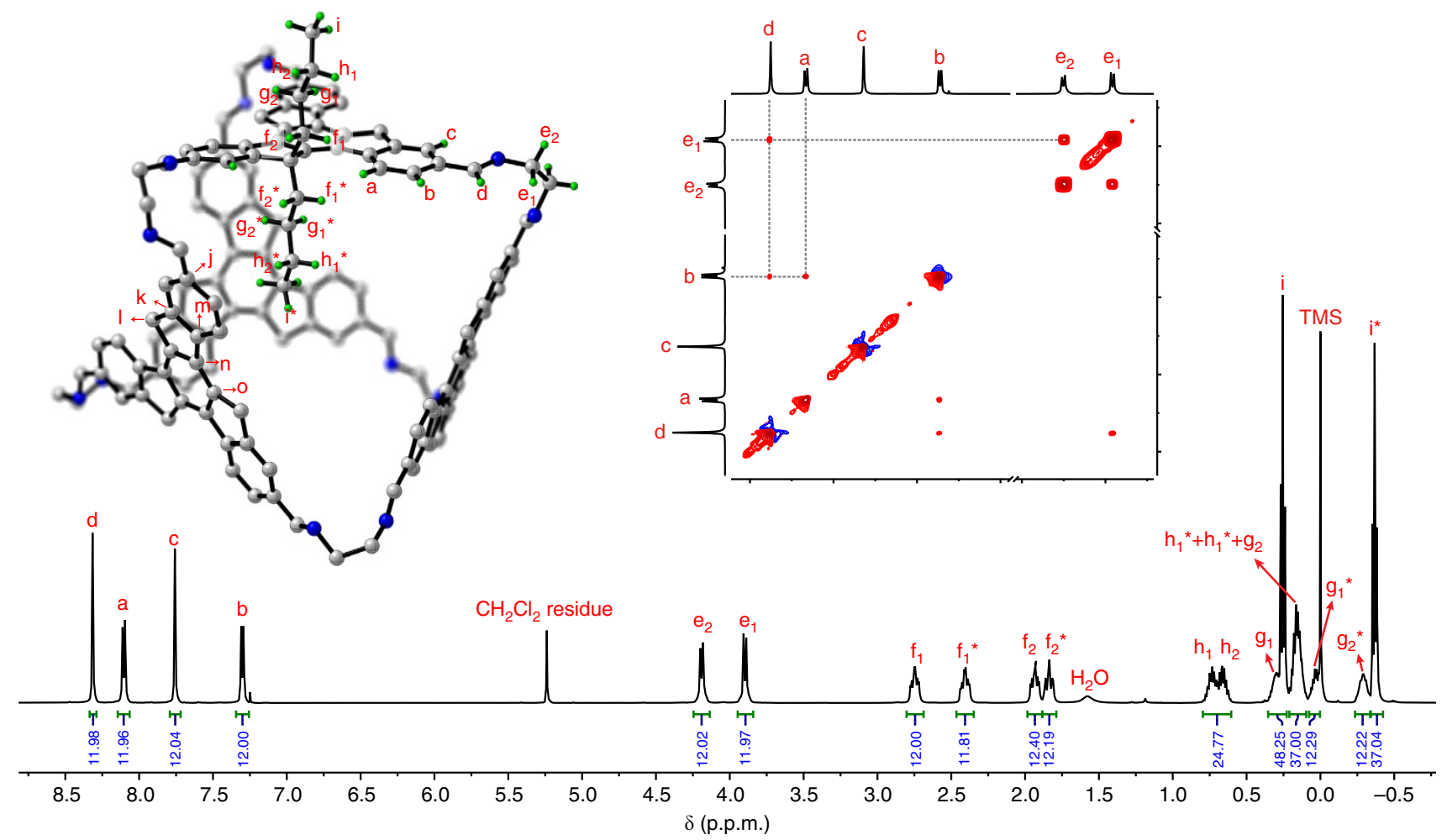

Figure $3 \mid{ }^{\mathbf{1}} \mathbf{H}$ NMR and NOE spectra of octahedron 1. ${ }^{1} \mathrm{H}$ NMR spectrum was measured in dichloromethane and fully rationalised by matching the crystal structure and 2D NMR spectra. The inserted NOE spectrum exhibits the crosspeaks of $\mathrm{H}^{\mathrm{d}}-\mathrm{H}^{\mathrm{b}}$ and $\mathrm{H}^{\mathrm{d}}-\mathrm{H}^{\mathrm{e}}$, suggesting $\mathrm{H}^{\mathrm{d}}$ is spatially close to $\mathrm{H}^{\mathrm{b}}$ and $\mathrm{H}^{\mathrm{e}}$ in solution, as presented in the molecular structure (only one butyl chain is shown for clarity).

and $(S, S)$-CHDA exhibit a torsion angle of $c a .60^{\circ}$. Therefore, we envisioned that the conformational preorganisation of diamines in $(R, R)$-CHDA or $(S, S)$-CHDA would dominate the facial directionality of the octahedra.

Under the same conditions as those used to prepare $\mathbf{1}$, $(R, R)$-CHDA and TR were reacted at room temperature for $24 \mathrm{~h}$. The mass spectrum of the product suggested the exclusive formation of $\mathrm{TR}_{4} \mathrm{CHDA}_{6}$ with a M.W. of 3520.52 (calculated M.W.: 3520.59), corresponding to octahedron 2. However, the ${ }^{1} \mathrm{H}$ NMR spectrum exhibited eleven signals for the imine protons $\left(\mathrm{H}^{\mathrm{d}}\right)$, indicating a mixture of diastereomeric octahedra with all three symmetries, $T, C_{3}$ and $C_{2}$ (Supplementary Fig. 11a). HPLC analysis revealed a distribution of three diastereomers, which eluted at $10.3 \mathrm{~min}(42 \%), 14.9 \mathrm{~min}(42 \%)$ and $24.1 \mathrm{~min}(14 \%)$ (Fig. 4a). We isolated each diastereomer and determined its symmetry through the number of imine signals in the NMR spectra. The $T-, C_{3^{-}}$, and $C_{2^{-}}$symmetric diastereomers that eluted at $10.3,14.9$, and $24.1 \mathrm{~min}$ gave rise to a single $\mathrm{H}^{\mathrm{d}}$ signal, four $\mathrm{H}^{\mathrm{d}}$ signals and six $\mathrm{H}^{\mathrm{d}}$ signals, respectively (Supplementary Figs 11-14). The $C_{2}$-symmetric diastereomer (CCAA)-2 partially converted into other diastereomers after $24 \mathrm{~h}$, thus suggesting that it is a kinetic product. To convert all kinetic products to their corresponding thermodynamic products, we heated 2 at $110^{\circ} \mathrm{C}$ for $48 \mathrm{~h}$ after stirring at room temperature for $24 \mathrm{~h}$. HPLC and NMR analyses indicated the near quantitative transformation to the $T$-symmetric diastereomer, which eluted at $11.2 \mathrm{~min}$ (Fig. 4a and Supplementary Fig. 11b).

On the basis of single-crystal X-ray diffraction analysis, we confirmed the $T$ - and $C_{3}$-symmetric diastereomers to be (AAAA)-2 and (CAAA)-2 octahedra, respectively (Fig. 4c,d). The chirality of the octahedra was inherited from that of the $(R, R)$-CHDA vertices. As in $(A A A A)-\mathbf{1}$, all imine bonds in $(A A A A)-2$ and the $A$ faces of $(C A A A)-2$ rotate towards the $\mathrm{sp}^{3}$ carbons of truxene, whereas the $A$ face of $(A A A A)-2$ rotates a

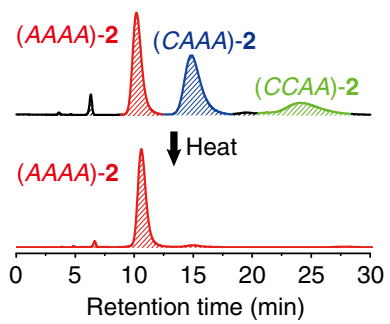

C

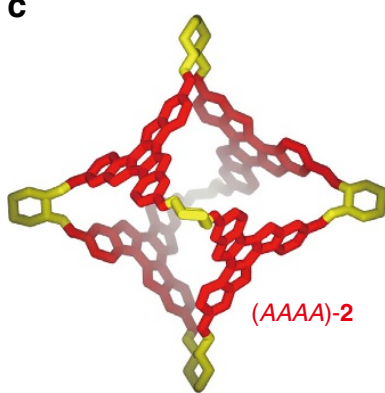

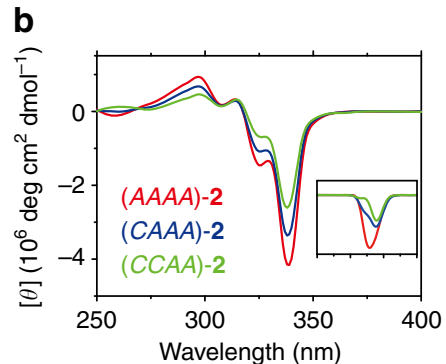

d

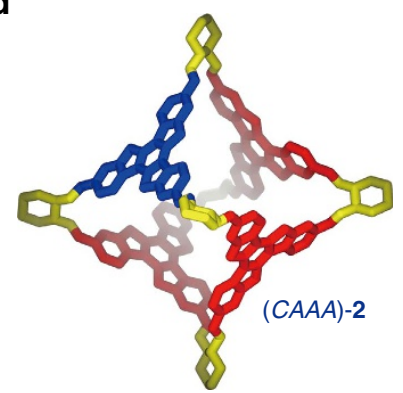

Figure 4 | Characterisation of octahedra 2. (a) Chiral HPLC spectra of the kinetic products of $\mathbf{2}$ synthesised at $25^{\circ} \mathrm{C}$ (top) revealing three diastereomers (i.e., $(A A A A)-\mathbf{2},(C A A A)-\mathbf{2}$ and $(C C A A)-\mathbf{2})$ that changed into the thermodynamic product $(A A A A)-2$ when heated at $110^{\circ} \mathrm{C}$ for $48 \mathrm{~h}$ (bottom). (b) Experimental and (ZINDO/S)-predicted (the insert) $C D$ spectra of ( $A A A A)-\mathbf{2}$ (red), (CAAA)-2 (blue) and (CCAA)-2 (green) in hexane. (c,d) Single-crystal structures of the thermodynamic (AAAA)-2 (c) and kinetically stable (CAAA)-2 (d) (hydrogen atoms and butyl groups are omitted for clarity). 
against the $\mathrm{sp}^{3}$ carbons to match the gauche conformation of the diamine vertex. This response in the imine arrangements to the directional change of the truxene faces is rare, presumably due to the rigidity in the diamine vertices, truxene skeletons and imine junctions.

The structural change in these diastereomers also leads to significantly different spectroscopic results. The $\mathrm{CD}$ spectra of $(A A A A)-2$, (CAAA)-2 and (CCAA)-2 are similar to but sequentially weaker than that of $(A A A A)$-1, consistent with the (ZINDO/S)-predicted CD spectra (Fig. $4 \mathrm{~b}$, insert). This trend can be rationalized by the decrease in the alternate angles of the truxene cores (Supplementary Fig. 15).

As expected, the enantiomeric vertex of $(S, S)$-CHDA led to enantiomeric formation of the thermodynamic octahedron (CCCC)-3 through the (CCCA)-3 and (CCAA)-3 kinetic octahedra (Supplementary Fig. 16).

Kinetics of the synthesis of FRP 2. The formation of octahedron 2 at $298 \mathrm{~K}$ constitutes a kinetically controlled dynamic combinatorial library 6,46 . Therefore, we monitored the library, which contained $1.6 \mathrm{mM}$ TR, by time-dependent chiral-HPLCMS, UV-vis and CD to investigate the possible mechanistic pathways ${ }^{6,52,53}$ (Fig. 5). In $5 \mathrm{~min}$, most of the $\mathbf{T R}$ reacted with diamine to form the $[1+1]$ and $[1+2]$ intermediates, which gave rise to a negligible $\mathrm{CD}$ signal. In $25 \mathrm{~min}$, the reaction generated more $[3+3],[3+4]$, and $[4+5]$ intermediates, which exhibited a strong CD signal. All intermediates disappeared at $60 \mathrm{~min}$ except for the $[4+5]$ intermediate, which gradually transformed into the $[4+6]$ octahedra after $24 \mathrm{~h}$. At room temperature, the formation and cleavage of an imine bond is fast but the transformation from the kinetic (CAAA)-2 and (CCAA)-2 to the thermodynamic $(A A A A)-2$ was extremely slow. Therefore, we postulated that the cyclohexyl group of CHDA shields the imine bonds in the octahedral 'testudo formation' in a cooperative manner.
Reasons for the facial homo-directionality in FRP 1 and 2. To shed light on the homo-directionality of the CCCC or AAAA configuration of all thermodynamic products of octahedra 1, 2 and 3, we calculated the free energies of different stereoisomers by the Compass II force field. The (CCCC)-1 and (AAAA)-1 enantiomers had the lowest energies among all stereoisomers of $\mathbf{1}$, whereas the enantiomeric production of $(A A A A)-\mathbf{2}$ and (CCCC)-3 can be rationalized by their relative energies being the lowest among all stereoisomers of $\mathbf{2}$ and $\mathbf{3}$ (Table 1). In addition, the energy differences among the diastereomers were primarily due to non-covalent contacts and repulsion forces of the butyl groups inside the octahedra. For illustration, the energy of $(A A A A)-1$ was calculated to be $12.2 \mathrm{kcal} \mathrm{mol}^{-1}$ lower than that of $(C A A A)-1$, in which $9.7 \mathrm{kcal} \mathrm{mol}^{-1}$ was attributed to the difference in van de Waals interaction. This phenomenon is correlated to the crowded interior of the octahedra in the crystal structures, as well as in the simulated structures (Supplementary Fig. 17). Therefore, we speculate that the non-covalent repulsion forces among the interior butyl groups are essential for directing the homo-directional facial rotations of the thermodynamic polyhedra.

\section{Discussion}

We introduced 2D chiral truxene building block to generate a series of 3D chiral polyhedra with rotational faces. The homo-directionality in our thermodynamic polyhedra resembles the identical rotational directionality of each facial unit in some virus capsids. By controlling the vertex, we stereoselectively synthesised thermodynamic homo-directional FRP and kinetic hetero-directional products. This strategy could be extended to other multifarious 2D asymmetric molecules to produce more complex Platonic and Archimedean polyhedra, which could be used for molecular recognition, chiral separation or asymmetric catalysis. We anticipate our assay to be a starting point towards
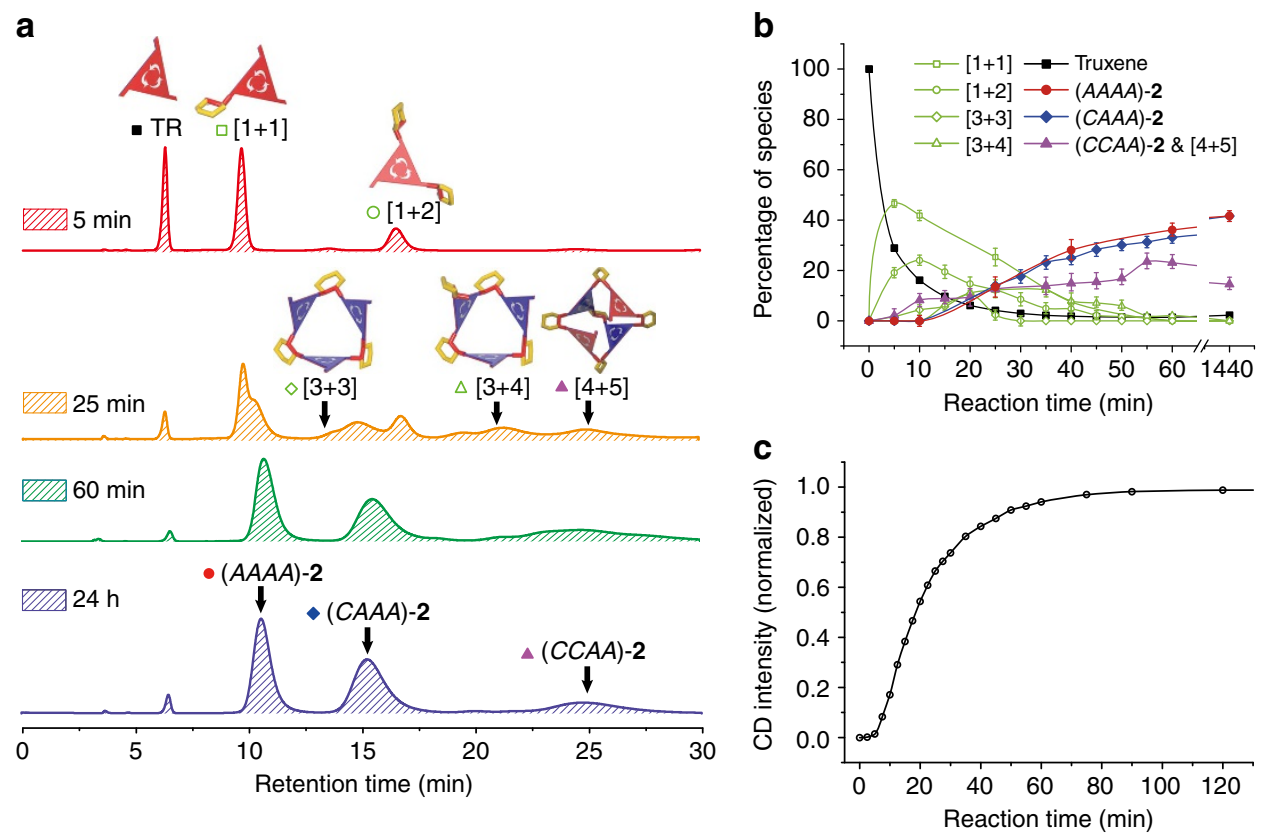

Figure 5 | Time-dependent characterisation of the synthesis of octahedron 2. (a) Selected HPLC spectra at certain time intervals. The components of the intermediates were determined by mass spectrometry. (b) Kinetic profile of the synthesis of octahedron $\mathbf{2}$ (1.6 mM at $298 \mathrm{~K}$, absorption monitored at $325 \mathrm{~nm}$ ), showing the consumption of truxene and the formation of three octahedral diastereomers. The formation and disappearance of the intermediates with the $[1+1],[1+2],[3+3],[3+4]$ and $[4+5]$ components can also be tracked. The percentages were evaluated from the HPLC peak areas. (c) Timedependent $C D$ intensities at $340 \mathrm{~nm}$ normalised by the intensity at $24 \mathrm{~h}$. 
Table 1 | Energies of octahedra 1 and 2 calculated with COMPASS II force field.

\begin{tabular}{|c|c|c|c|c|c|c|}
\hline \multirow{2}{*}{$\begin{array}{l}\text { Structure }^{\star} \\
(C C C C)-1\end{array}$} & \multirow{2}{*}{$\begin{array}{c}\begin{array}{c}\text { Valence energy } \\
\text { (diag. terms) }\end{array} \\
726.711\end{array}$} & \multirow{2}{*}{$\begin{array}{c}\begin{array}{c}\text { Valence energy } \\
\text { (cross terms) }\end{array} \\
-97.164\end{array}$} & \multicolumn{3}{|c|}{ Non-bond energy ${ }^{\S}=($ van der Waals + electrostatic $)$} & \multirow{2}{*}{$\begin{array}{c}\text { Total energy } \\
668.112\end{array}$} \\
\hline & & & 38.566 & 3.093 & 35.473 & \\
\hline$(C C C A)-\mathbf{1}$ & 731.605 & -98.323 & 47.061 & 12.828 & 34.233 & 680.343 \\
\hline$(C A A A)-\mathbf{1}$ & 731.607 & -98.324 & 47.060 & 12.826 & 34.234 & 680.343 \\
\hline$(A A A A)-\mathbf{1}$ & 726.717 & -97.163 & 38.558 & 3.087 & 35.471 & 668.112 \\
\hline$(C C A A)-\mathbf{2}$ & 650.221 & -121.005 & 63.485 & 28.970 & 34.516 & 592.702 \\
\hline$(C A A A)-\mathbf{2}$ & 649.340 & -121.103 & 57.521 & 19.378 & 38.143 & 585.757 \\
\hline$(A A A A)-\mathbf{2}$ & 641.876 & -118.330 & 44.825 & 8.590 & 36.235 & 568.371 \\
\hline$(C C C C)-\mathbf{3}$ & 641.875 & -118.331 & 44.826 & 8.591 & 36.235 & 568.371 \\
\hline$(C C C A)-\mathbf{3}$ & 647.413 & -119.395 & 53.218 & 17.367 & 35.851 & 581.236 \\
\hline
\end{tabular}

*Structures were constructed on the basis of the crystal structures and adjusted to follow the symmetries of $T$ (CCCC and $A A A A), C_{3}(C C C A$ and $C A A A)$ and $C_{2}$ (CCAA). The constructed structures were calculated with the COMPASS II force field.

Valence energy (diag. terms) contains the contributions of bond, angle, torsion, and inversion.

¥Valence energy (cross terms) contains the contributions of stretch-stretch, stretch-bend-stretch, stretch-torsion-stretch, separated-stretch-stretch, torsion-stretch, bend-bend, torsion-bend-bend and bend-torsion-bend.

$\S$ Non-bond energy contains the contributions of van der Waals and electrostatic interactions.

"All of the energies are reported in $\mathrm{kcal} \mathrm{mol}^{-1}$. The differences among total energies of each isomers are mostly rooted in the differences in van der Waals interaction, as highlighted in two bold entries.

more sophisticated molecular polyhedra with rotational faces, providing a strategy to mimic the sophistication of viruses.

\section{Methods}

Synthesis of the FRP. Vicinal diamine (6 eq) in anhydrous toluene and TFA (0.025 eq) were added to a schlenk flask (1 l ) and stirred until dissolved. TR (4 eq) in toluene ( 400 to $600 \mathrm{ml}$ ) was added dropwise to the flask within $24 \mathrm{~h}$. The solution was kept stirring for another $24 \mathrm{~h}$. Solvents were evaporated under vacuum and solid was used for chiral separation and crystal growth without further purification. General experimental information and characterization details can be found in the Supplementary Methods.

NMR and MS characterization. ${ }^{1} \mathrm{H}$ and ${ }^{13} \mathrm{C}$ nuclear magnetic resonance (NMR) spectra were recorded on a Bruker AVIII-500 spectrometer $(500$ and $125 \mathrm{MHz}$, respectively) or Bruker AVIII-850 spectrometer at $298 \mathrm{~K}$ ( 850 and $213 \mathrm{MHz}$, respectively) and are reported relative to residual solvent signals. Matrix-assisted laser desorption/ionization time of flight mass spectra (MALDI-TOF MS) were collected on a Bruker microflex LT-MS with 2,4,6-trihydrotyacetophenane $(0.05 \mathrm{M}$ in methanol) as matrix. High-resolution mass spectra (HRMS) were collected on a Bruker En Apex Ultra 7.0T FT-MS.

Single-crystal X-ray diffraction. Single crystal X-ray diffraction data were collected on Rigaku SuperNova X-Ray single crystal diffractometer using $\mathrm{Cu} \mathrm{K} \alpha$ $(\lambda=1.54184 \AA)$ micro-focus X-ray sources at $100 \mathrm{~K}$. The raw data were collected and reduced by CrysAlisPro software, the structures were solved by SHELXS or SHELXT and refined by SHELXL. Solution and refinement procedures are presented in the Supplementary Methods and specific details are compiled in Supplementary Table 1 and Supplementary Figs 18-22. Crystallographic information files are provided as Supplementary Data (Supplementary Data 1 for $\mathbf{1}$, Supplementary Data 2 for $(A A A A)$-2, Supplementary Data 3 for $(C A A A)-2$, Supplementary Data 4 for $(C C C C)-3$, Supplementary Data 5 for $(C C C A)-3)$.

HPLC and CD characterization. HPLC analyses were performed on a Shimadzu LC-16A instrument, using Daicel Chiralcel IE Columns. FRP 1 was separated by HPLC in the mobile phase of hexane:ethyl acetate $=83: 17(\mathrm{v} / \mathrm{v})$ with $0.1 \%$ diethylamine, and FRP 2 was separated in the mobile phase of hexane:ethanol $=75: 25(\mathrm{v} / \mathrm{v})$ with $0.1 \%$ diethylamine. $\mathrm{CD}$ spectra were collected on JASCO J-810 circular dichroism spectrometer at $298 \mathrm{~K}$. All CD spectra of the separated enantiomers were measured in hexane.

Kinetics of the synthesis. TR (122 mg, $0.160 \mathrm{mmol}),(\mathrm{R}, \mathrm{R})-\mathrm{CHDA}(14.4 \mathrm{mg}$, $0.240 \mathrm{mmol})$ and TFA $(1.37 \mathrm{mg}, 0.0120 \mathrm{mmol})$ were reacted in toluene $(100 \mathrm{ml})$ at $298 \mathrm{~K}$. At certain time intervals, the reaction solution were drawn out and measured by HPLC in the mobile phase of hexane:ethanol $=75: 25(\mathrm{v} / \mathrm{v})$ with $0.1 \%$ diethylamine. The experimental details are presented in the Supplementary Methods and HPLC spectra are compiled in Supplementary Figs 23-35.

Computational methods. All the structural optimization and energy calculation were carried out in Materials Studios 7.0 by using the COMPASS II force field. The CD spectra were calculated at ZINDO semi-empirical level with Gaussian 09. Details of the method are provided in the Supplementary Methods. Coordinates of all optimized structures are provided in Supplementary Data 6.

Data availability. Crystallographic data in this study were deposited at the Cambridge Crystallographic Data Centre with the accession codes (CCDC 1063965, 1406534, 1406540, 1406657, and 1407221). The authors declare that all other data supporting the findings of this study are available within the article and its Supplementary Information files or available from the authors upon reasonable request.

\section{References}

1. Kroto, H. W., Heath, J. R., Obrien, S. C., Curl, R. F. \& Smalley, R. E. C60: Buckminsterfullerene. Nature 318, 162-163 (1985).

2. Dietrich-Buchecker, C. O. \& Sauvage, J.-P. A synthetic molecular trefoil knot. Angew. Chem. Int. Ed. 28, 189-192 (1989).

3. Conn, M. M. \& Rebek, J. Self-assembling capsules. Chem. Rev. 97, 1647-1668 (1997).

4. Chichak, K. S. et al. Molecular Borromean rings. Science 304, 1308-1312 (2004).

5. Forgan, R. S., Sauvage, J.-P. \& Stoddart, J. F. Chemical topology: complex molecular knots, links, and entanglements. Chem. Rev. 111, 5434-5464 (2011).

6. Ponnuswamy, N., Cougnon, F. B., Clough, J. M., Pantoş, G. D. \& Sanders, J. K. Discovery of an organic trefoil knot. Science 338, 783-785 (2012).

7. Ayme, J.-F., Beves, J. E., Campbell, C. J. \& Leigh, D. A. Template synthesis of molecular knots. Chem. Soc. Rev. 42, 1700-1712 (2013).

8. Lee, S., Chen, C.-H. \& Flood, A. H. A pentagonal cyanostar macrocycle with cyanostilbene $\mathrm{CH}$ donors binds anions and forms dialkylphosphate [3]rotaxanes. Nature Chem 5, 704-710 (2013).

9. Smulders, M. M. J., Riddell, I. A., Browne, C. \& Nitschke, J. R. Building on architectural principles for three-dimensional metallosupramolecular construction. Chem. Soc. Rev. 42, 1728-1754 (2013).

10. Fuller, R. B. Synergetics: Explorations In The Geometry Of Thinking (Macmillan, 1975).

11. Cromwell, P. R. Polyhedra (Cambridge Univ. Press, 1997).

12. Klug, A. \& Caspar, D. L. The structure of small viruses. Adv. Virus Res. 7, 225-325 (1960). 
13. Rossmann, M. G. \& Johnson, J. E. Icosahedral RNA virus structure. Annu. Rev. Biochem. 58, 533-573 (1989).

14. Tate, J. et al. The crystal structure of cricket paralysis virus: the first view of a new virus family. Nature Struct. Biol. 6, 765-774 (1999).

15. Prasad, B. V. \& Schmid, M. F. Principles of virus structural organization. Adv. Exp. Med. Biol. 726, 17-47 (2012).

16. MacGillivray, L. R. \& Atwood, J. L. A chiral spherical molecular assembly held together by 60 hydrogen bonds. Nature 389, 469-472 (1997).

17. Nuckolls, C., Hof, F., Martín, T. \& Rebek, J. Chiral microenvironments in self-assembled capsules. J. Am. Chem. Soc. 121, 10281-10285 (1999).

18. Prins, L. J., De Jong, F., Timmerman, P. \& Reinhoudt, D. N. An enantiomerically pure hydrogen-bonded assembly. Nature 408, 181-184 (2000).

19. Cook, T. R., Zheng, Y.-R. \& Stang, P. J. Metal-organic frameworks and self-assembled supramolecular coordination complexes: Comparing and contrasting the design, synthesis, and functionality of metal-organic materials. Chem. Rev. 113, 734-777 (2013).

20. Sun, Q. F. et al. Self-assembled $\mathrm{M}_{24} \mathrm{~L}_{48}$ polyhedra and their sharp structural switch upon subtle ligand variation. Science 328, 1144-1147 (2010).

21. Yoshizawa, M., Tamura, M. \& Fujita, M. Diels-alder in aqueous molecular hosts: unusual regioselectivity and efficient catalysis. Science 312, 251-254 (2006).

22. Liu, Y., Hu, C., Comotti, A. \& Ward, M. D. Supramolecular Archimedean cages assembled with 72 hydrogen bonds. Science 333, 436-440 (2011).

23. Olenyuk, B., Whiteford, J. A., Fechtenkotter, A. \& Stang, P. J. Self-assembly of nanoscale cuboctahedra by coordination chemistry. Nature 398, 796-799 (1999).

24. Zhang, G., Presly, O., White, F., Oppel, I. M. \& Mastalerz, M. A permanent mesoporous organic cage with an exceptionally high surface area. Angew. Chem. Int. Ed. 53, 1516-1520 (2014).

25. Cook, T. R., Vajpayee, V., Lee, M. H., Stang, P. J. \& Chi, K.-W. Biomedical and biochemical applications of self-assembled metallacycles and metallacages. Acc. Chem. Res. 46, 2464-2474 (2013).

26. Slater, A. G. \& Cooper, A. I. Function-led design of new porous materials. Science 348, aaa8075 (2015).

27. Zhang, G. \& Mastalerz, M. Organic cage compounds from shape-persistency to function. Chem. Soc. Rev. 43, 1934-1947 (2014).

28. Zarra, S., Wood, D. M., Roberts, D. A. \& Nitschke, J. R. Molecular containers in complex chemical systems. Chem. Soc. Rev. 44, 419-432 (2015).

29. Ward, M. D. \& Raithby, P. R. Functional behaviour from controlled self-assembly: challenges and prospects. Chem. Soc. Rev. 42, 1619-1636 (2013).

30. Liu, T., Liu, Y., Xuan, W. \& Cui, Y. Chiral nanoscale metal-organic tetrahedral cages: Diastereoselective self-assembly and enantioselective separation. Angew. Chem. Int. Ed. 49, 4121-4124 (2010).

31. Li, K. et al. Stepwise assembly of $\mathrm{Pd}_{6}\left(\mathrm{RuL}_{3}\right)_{8}$ nanoscale rhombododecahedral metal-organic cages via metalloligand strategy for guest trapping and protection. J. Am. Chem. Soc. 136, 4456-4459 (2014).

32. Bolliger, J. L., Belenguer, A. M. \& Nitschke, J. R. Enantiopure water-soluble $\left[\mathrm{Fe}_{4} \mathrm{~L}_{6}\right]$ cages: host-guest chemistry and catalytic activity. Angew. Chem. Int. Ed. 52, 7958-7962 (2013).

33. Tozawa, T. et al. Porous organic cages. Nature Mater 8, 973-978 (2009).

34. Xu, D. \& Warmuth, R. Edge-directed dynamic covalent synthesis of a chiral nanocube. J. Am. Chem. Soc. 130, 7520-7521 (2008).

35. Olson, A. J., Hu, Y. H. \& Keinan, E. Chemical mimicry of viral capsid self-assembly. Proc. Nat. Acad. Sci. USA 104, 20731-20736 (2007)

36. Narasimhan, S. K., Lu, X. \& Luk, Y. Y. Chiral molecules with polyhedral T, O, or I symmetry: theoretical solution to a difficult problem in stereochemistry. Chirality 20, 878-884 (2008).

37. Cook, T. R. \& Stang, P. J. Recent developments in the preparation and chemistry of metallacycles and metallacages via coordination. Chem. Rev. 115, 7001-7045 (2015).

38. Zhao, C. et al. Chiral amide directed assembly of a diastereo- and enantiopure supramolecular host and its application to enantioselective catalysis of neutral substrates. J. Am. Chem. Soc. 135, 18802-18805 (2013).

39. Xie, T.-Z. et al. Construction of a highly symmetric nanosphere via a one-pot reaction of a tristerpyridine ligand with $\mathrm{Ru}(\mathrm{ii})$. J. Am. Chem. Soc. 136, 8165-8168 (2014)

40. Caulder, D. L. et al. Design, formation and properties of tetrahedral $\mathrm{M}_{4} \mathrm{~L}_{4}$ and $\mathrm{M}_{4} \mathrm{~L}_{6}$ supramolecular clusters. J. Am. Chem. Soc. 123, 8923-8938 (2001).

41. Han, M., Engelhard, D. M. \& Clever, G. H. Self-assembled coordination cages based on banana-shaped ligands. Chem. Soc. Rev. 43, 1848-1860 (2014).

42. Castilla, A. M., Ramsay, W. J. \& Nitschke, J. R. Stereochemistry in subcomponent self-assembly. Acc. Chem. Res. 47, 2063-2073 (2014).

43. Meng, W. J., Ronson, T. K. \& Nitschke, J. R. Symmetry breaking in selfassembled $\mathrm{M}_{4} \mathrm{~L}_{6}$ cage complexes. Proc. Nat. Acad. Sci. USA 110, 10531-10535 (2013).
44. Belowich, M. E. \& Stoddart, J. F. Dynamic imine chemistry. Chem. Soc. Rev. 41, 2003-2024 (2012).

45. Rowan, S. J., Cantrill, S. J., Cousins, G. R., Sanders, J. K. \& Stoddart, J. F. Dynamic covalent chemistry. Angew. Chem. 41, 898-952 (2002).

46. Lehn, J.-M. From supramolecular chemistry towards constitutional dynamic chemistry and adaptive chemistry. Chem. Soc. Rev. 36, 151-160 (2007).

47. Goubard, F. \& Dumur, F. Truxene: a promising scaffold for future materials. RSC Adv. 5, 3521-3551 (2015).

48. Gomez-Lor, B., de Frutos, O., Ceballos, P. A., Granier, T. \& Echavarren, A. M. Synthesis of new $C_{3 \mathrm{~h}}$ and $C_{3 \mathrm{v}}$ truxene derivatives. Eur. J. Org. Chem 2107-2114 (2001).

49. Meng, W., Clegg, J. K., Thoburn, J. D. \& Nitschke, J. R. Controlling the transmission of stereochemical information through space in terphenyl-edged $\mathrm{Fe}_{4} \mathrm{~L}_{6}$ cages. J. Am. Chem. Soc. 133, 13652-13660 (2011).

50. Borzsonyi, G. et al. Water-soluble J-type rosette nanotubes with giant molar ellipticity. J. Am. Chem. Soc. 132, 15136-15139 (2010).

51. Yang, Y., da Costa, R. C., Fuchter, M. J. \& Campbell, A. J. Circularly polarized light detection by a chiral organic semiconductor transistor. Nature Photon 7, 634-638 (2013).

52. Kang, J. et al. A rational strategy for the realization of chain-growth supramolecular polymerization. Science 347, 646-651 (2015).

53. Korevaar, P. A. et al. Pathway complexity in supramolecular polymerization. Nature 481, 492-496 (2012).

\section{Acknowledgements}

We thank Jean-Marie Lehn, E.W. (Bert) Meijer, Jay S. Siegel, Stephen Z. D. Cheng, Kenneth N. Raymond, Takuzo Aida, Anthony Judge, Jian Pei, Xi Zhang, Minghua Liu, Haibo Yang, Yiqin Gao, Fuji Zhang, Yunbao Jiang, Zhaoxiong Xie, Chengyong Su, Jinliang Wang, Wei Zhang, Xiaoye Wang, Ting Lei, Cheng Wang, Tingbin Wen, Gang Fu, Yuanzhi Tan, Bin Ren, Chaoyong (James) Yang, Erqiang Chen and Haiping Xia for discussions. We also thank Wei Xuan, Ming Luo, Zier Yan, Jijun Jiang, Zhangwen Wei, Zhixin Chen, Ziang Nan and Lei Gong for assistance in experiments. Cover art was helped by Zhuoru Li. This work is supported by the National Science Foundation of China (Nos. 91427304, 21573181, 91227111 and 21102120), the 973 Program (No. 2015CB856500), the Beijing National Laboratory for Molecular Science (No. 20140114), the Fundamental Research Funds for the Central Universities (No. 20720160050) of China and Program for Changjiang Scholars and Innovative Research Team in University. This work is in memorial of Zhengluo Cao for his science mentorship to Xiaoyu Cao and his contribution during the early stages of this project.

\section{Author contributions}

X.C., X.W. and Y.W. conceived, initiated and developed this work. X.W. synthesised the building blocks and the polyhedra. X.W. and Y.W. performed NMR, MS and XRD analyses. Y.W. performed the HPLC and CD analyses. Y.W. carried out the characterisation of kinetics and performed the calculations. All authors participated in analysis and interpretation of the results. Y.W. drafted the manuscript, with contributions from X.W., X.C. and Z.T. X.C., Y.W. and X.W. coordinated the efforts of the research teams.

\section{Additional information}

Supplementary Information accompanies this paper at http://www.nature.com/ naturecommunication

Competing financial interests: The authors declare no competing financial interests.

Reprints and permission information is available online at http://npg.nature.com/ reprintsandpermissions/

How to cite this article: Wang, X. et al. Assembled molecular face-rotating polyhedra to transfer chirality from two to three dimensions. Nat. Commun. 7:12469 doi: 10.1038/ncomms12469 (2016).

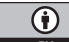

This work is licensed under a Creative Commons Attribution 4.0 International License. The images or other third party material in this article are included in the article's Creative Commons license, unless indicated otherwise in the credit line; if the material is not included under the Creative Commons license, users will need to obtain permission from the license holder to reproduce the material. To view a copy of this license, visit http://creativecommons.org/licenses/by/4.0/

(C) The Author(s) 2016 